\title{
Modeling Time-to- Recovery of Adult Diabetic Patients Using Cox-Proportional Hazards Model
}

\author{
Abiyot Negash Terefe, Assaye Belay Gelaw \\ Department of Statistics, College of Natural and Computational Science, Mizan-Tepi University, Tepi, Ethiopia \\ Email address: \\ abiyotnegash@yahoo.com (A. N. Terefe), asayestat@gmail.com (A. B. Gelaw)
}

To cite this article:

Abiyot Negash Terefe, Assaye Belay Gelaw. Modeling Time-to- Recovery of Adult Diabetic Patients Using Cox-Proportional Hazards Model. International Journal of Statistical Distributions and Applications. Vol. 3, No. 4, 2017, pp. 67-71. doi: 10.11648/j.ijsd.20170304.12

Received: August 21, 2017; Accepted: September 7, 2017; Published: November 10, 2017

\begin{abstract}
Diabetes is a group of diseases marked by high or low level of glucose resulting from defects in insulin production, insulin action or both. The objective of this study is to model time-to-first recovery of adult diabetic patients using Cox Proportional Hazards model. A retrospective data was obtained from Jimma University Specialized Hospital diabetic patient clinic whose age $\geq 18$ years and under treatments in between September 2010 and August 2013 are included in the study. Time of fasting blood sugar level to reach the first normal range, $70-130 \mathrm{mg} / \mathrm{dl}$ of blood were the response variable. Cox Proportional Hazard model were used. Types of diabetic, bodyweight at baseline, fasting blood sugar at baseline, sex and age of patients are significantly associated with time to first recovery of diabetic patients. These variables are important factors that should be considered during the selection phase a treatment (combination of treatments) for diabetes.
\end{abstract}

Keywords: Akaike's Information Criterion, Cox-Snell, Deviance Residuals

\section{Introduction}

Diabetes is a group of diseases marked by high or low level of glucose resulting from defects in insulin production, insulin action or both. It can lead to serious complication and premature death but steps to control the disease and lowers the risk of complications does exist [1]. Diabetes is becoming one of the rapidly increasing non-communicable diseases and an important public health problem all over the world. The main factors which lead to the cause of diabetes mellitus are hereditary (genetics) [2].

382 million people have diabetes in 2013; by 2035 this will rise to 592 million. The number of people with type 2 diabetes is increasing in every country. $80 \%$ of people with diabetes live in low- and middle-income countries. The greatest number of people with diabetes is between 40 and 59 years of age. Ethiopia is one of the developing countries where by the prevalence is increasing time to time. The prevalence of diabetes in Ethiopia for 20-79 age groups in 2013 is 4.89 , Uganda 4.8 [3]. Recommended blood sugar for people with diabetes (according to the WHO) before meals plasma glucose levels within a narrow range $70-130 \mathrm{mg} / \mathrm{dl}$ (milligram per deciliter). Patients from three hospital in Uganda, time to remission was found to decrease with increase in body mass index and age. Males tend to recover faster than the female and the less or non-educated controlled the disease better than the educated ones [4].

All patients attending the diabetic clinic at Mekelle Hospital, their median age and diabetes duration were 30 and 5 years, respectively, with a male excess of $2: 1$. Median BMI was $20.6 \mathrm{~kg} / \mathrm{m}^{2}$. Despite these clinical characteristics suggestive of type 1 diabetes, only 42 of 69 (61\%) patients were C-peptide- negative and 35\% GADA-positive [5].

$\mathrm{DM}$ is a life-long challenge that requires behavioral change and adequate self-care practices for better glycaemic control. This disease has tendency to cause death mostly when its co-infection manifested on patient with other disease like AIDs, heart diseases, stroke and others. It has high chance of making a patient economically dependent on their family/household members rather than the loss it can cause from his/her none productiveness. As economic development starts from individual contribution it needs to overcome the effect of DM on health of individuals in the society [6]. Hence, it needs to identify factors considerably related to it, which intern assists to give due attention to these problems so that we can prolong the life of DM patients. 


\section{Methods}

Longitudinal retrospective cohort follow up of adult diabetic patients data is collected from JUSH Diabetic Patient Clinic located in southwest of Ethiopia. The data is extracted from the patient's chart which contains epidemiological, laboratory and clinical information of all diabetic patients under insulin treatment follow-up. A total of 1930 diabetic patients are on active follow up. All diabetic patients greater than or equal to 18 years old and placed under treatments that have followed between September 2010 and August 2013 (three years data) were included. The data for this study consists of 544 individuals.
The outcome variable considered in this study is the time to first recovery of diabetic patients until it reaches normal fasting (before meal) blood sugar level in the follow up period. Time to first recovery means the time until patients comes to normal fasting blood sugar level for first time in the follow up period according to WHO scale (70-130mg/dl). Right censoring is considered when patient is not recovered once between the study time, transferred to other hospital and death before first recovery to normal blood sugar level. Covariates considered are Sex, Age (years), Diabetic type, Family history, systolic and diastolic blood pressure in $\mathrm{mm} / \mathrm{hg}$, Bodyweight in $\mathrm{kg}$, and fasting blood sugar in $\mathrm{mg} / \mathrm{dl}$.

Table 1. Diabetes mellitus patient baseline covariates of median recovery, percentage and frequencies.

\begin{tabular}{|c|c|c|c|c|c|}
\hline \multirow[b]{2}{*}{ Covariates } & & \multicolumn{3}{|c|}{ Type-I Diabetic Mellitus (DM) } & \multirow[b]{2}{*}{ 95\% CI, Median } \\
\hline & & Total (\%) & Recovery (\%) & Median & \\
\hline \multirow{2}{*}{ Sex } & Male & $84(23.93)$ & $71(84.52)$ & 2 & $(2,3)$ \\
\hline & Female & $37(19.17)$ & $32(86.49)$ & 1 & $(1,2)$ \\
\hline \multirow{2}{*}{ Family history } & Yes & $121(22.24)$ & $103(85.12)$ & 2 & $(1,2)$ \\
\hline & No & & & & \\
\hline \multirow{5}{*}{ Age at baseline (yrs) } & $<30$ & $39(35.78)$ & $38(97.44)$ & 1 & $(1,2)$ \\
\hline & $30-44$ & $53(34.19)$ & $44(83.02)$ & 2 & $(2,3)$ \\
\hline & $45-59$ & $22(13.25)$ & $19(86.36)$ & 2 & $(1,2)$ \\
\hline & $60-74$ & $4(4.6)$ & $2(50)$ & 4 & \\
\hline & $>74$ & $3(11.11)$ & 0 & & \\
\hline \multirow{3}{*}{$\mathrm{SBP}(\mathrm{mm} / \mathrm{Hg})$} & $<110$ & $19(20.21)$ & $18(94.74)$ & 1 & $(1,2)$ \\
\hline & $110-130$ & $84(24.63)$ & $71(84.52)$ & 2 & $(1,2)$ \\
\hline & $>130$ & $18(16.51)$ & $14(77.78)$ & 2 & $(1,2)$ \\
\hline \multirow{3}{*}{$\mathrm{DBP}(\mathrm{mm} / \mathrm{Hg})$} & $<60$ & $2(25)$ & $2(100)$ & 1 & \\
\hline & $60-80$ & $101(23.88)$ & $86(85.15)$ & 2 & $(1,2)$ \\
\hline & $>80$ & $18(15.93)$ & $15(83.33)$ & 1.5 & $(1,3)$ \\
\hline Overall & & $121(22.24)$ & $103(85.12)$ & 2 & $(1,2)$ \\
\hline Overall DM & & $404(74.265)$ & & 3 (Median) & $(3,4)$ \\
\hline
\end{tabular}

Table 1. Continued.

\begin{tabular}{|c|c|c|c|c|c|}
\hline \multirow[b]{2}{*}{ Covariates } & & \multicolumn{3}{|c|}{ Type-II Diabetic Mellitus (DM) } & \multirow[b]{2}{*}{ 95\% CI, Median } \\
\hline & & Total (\%) & Recovery (\%) & Median & \\
\hline \multirow{2}{*}{ Sex } & Male & $267(76.07)$ & $202(75.66)$ & 3 & $(2,4)$ \\
\hline & Female & $156(80.83)$ & $99(63.46)$ & 8 & $(6,9)$ \\
\hline \multirow{2}{*}{ Family history } & Yes & & & & \\
\hline & No & $423(77.76)$ & $301(71.16)$ & 4 & $(4,5)$ \\
\hline \multirow{5}{*}{ Age at baseline (yrs) } & $<30$ & $70(64.22)$ & $64(91.43)$ & 1 & $(1,2)$ \\
\hline & $30-44$ & $102(65.81)$ & $82(80.39)$ & 3 & $(2,4)$ \\
\hline & $45-59$ & $144(86.75)$ & $101(70.19)$ & 5 & $(3,6)$ \\
\hline & $60-74$ & $83(95.4)$ & $44(53.01)$ & 10 & $(7,13)$ \\
\hline & $>74$ & $24(88.89)$ & $10(41.67)$ & 16 & $(10,23)$ \\
\hline \multirow{3}{*}{$\mathrm{SBP}(\mathrm{mm} / \mathrm{Hg})$} & $<110$ & 75 (79.79) & $57(76)$ & 5 & $(2,7)$ \\
\hline & $110-130$ & $257(75.37)$ & $186(72.37)$ & 4 & $(3,5)$ \\
\hline & $>130$ & $81(74.31)$ & $58(71.60)$ & 5 & $(3,6)$ \\
\hline \multirow{2}{*}{$\mathrm{DBP}(\mathrm{mm} / \mathrm{Hg})$} & $<60$ & $6(75)$ & $4(66.67)$ & 5 & $(4, \mathrm{NA})$ \\
\hline & $>80$ & $95(84.07)$ & $70(73.68)$ & 4 & $(3,6)$ \\
\hline Overall & & $423(77.76)$ & $301(71.16)$ & 4 & $(4,5)$ \\
\hline Overall DM & & & & & \\
\hline
\end{tabular}

Area: Jimma University Specialized Hospital (JUSH); study time: between September 2010 and August 2013 (a three year data); Median: Median recovery time; DM: Diabetic Mellitus.

\section{Survival Analysis}

Survival analysis examines and models the time it takes for events to occur. We used Kaplan-Meir estimator and Cox $\mathrm{PH}$ model for the analysis and model building [7]. We also used logrank tests for comparison of survival functions. The Proportional Hazards Model: - It was used for multivariate analysis to identify factors associated with recovery from diabetic and Cox proportional hazards $(\mathrm{PH})$ model. The Cox 
proportional hazards $(\mathrm{PH})$ regression model [8] a broadly applicable and the most widely used method of survival analysis.

The Cox PH model (1) is a semi-parametric model where the baseline hazard $\alpha(t)$ is allowed to vary with time:

$$
\mathrm{h}_{i}=\mathrm{h}_{0}(t) \mu_{t}=\mathrm{h}_{0}(t) \exp \left(X_{i}^{T} \beta\right)
$$

Where, $\mathrm{h}_{0}(t)$, is the baseline hazard function; $X_{i}$ is a vector of covariates and $\beta$ is a vector of parameters for fixed effects. Parameter estimate $\beta$ refers to the increase in loghazard with a one unit increase for the continuous covariate.

AIC will be used for model comparison if models are nonnested whereas, likelihood ratio test will be used for the nested models and Newton Raphson will be used to solve the partial likelihood equations [9]. Parameter estimates will be obtained by Breslow approximation to partial likelihood. After fitting the models to a set of survival data, the adequacy of the fitted models to the survival data will be checked using Cox-Snell residuals and martingale residuals [10].

We used Hosmer and Lemeshow and Collett that recommend the procedure in variable selection, including all variables that are significant in the univariable analysis at the 20 to 25 percent level and also any other variables which are presumed to be clinically important to fit the initial multivariable model, [11] [12].

\section{Results}

Baseline categorical covariates are illustrated in Table 1. The data consists of 544 patients aged equal to or above 18 years old and placed under treatments that have followed between $1^{\text {st }}$ September 2010 and $30^{\text {th }}$ August 2013 (a three years data) at JUSH were included to find out their time to first recovery in to normal blood sugar level.

The 95\% confidence interval for the recovery time was between 3 and 4 months but it varies depending on the covariates included in the study. The majority of the cases, $423(77.76 \%)$ were type-II diabetic, 351 (64.52\%) were males, $423(77.76 \%)$ had no family history of the disease. The results further shows that the majority of the patients were first detected at the age of $45-59$ years $(30.51 \%)$ followed by age group 30-44 (28.49\%) and a few cases at age above 74 years $(4.96 \%)$. It can be seen that most of the cases had normal upper (systolic) (62.68\%) and lower (diastolic) $(77.76 \%)$ blood pressure.

The median recovery time for type-I and type-II diabetic were between 2 and 4 months respectively. In type-I diabetic from the total of 103 recovered patients, 71 (84.52\%) were males and median recovery time was 2 months whereas, 32 $(86.49 \%)$ were females and median recovery time 1 month and in type-II from the total of 423 recovered patients, 202 $(75.66 \%)$ were males and median recovery time 3 months whereas, $99(63.46 \%)$ were females and median recovery time 8 months. Majorities of females with type-I DM are recover to normal blood sugar level as compared to males and majorities of males with type-II DM were recover to normal blood sugar level as compared to females.

\subsection{Modeling Recovery Time for DM Inseparably}

Table 2. Multivariable Cox-PH model for the diabetic types inseparably.

\begin{tabular}{llll}
\hline Cox-PH Model for DM inseparably & & \\
\hline Covariates & $\begin{array}{l}\text { Hazard Ratio } \\
\text { (HR) }\end{array}$ & se(Coef $(\hat{\beta}))$ & P-value \\
\hline Age & & & \\
30-44 years & 0.679 & 0.135 & 0.004 \\
45-59 years & 0.499 & 0.139 & 0.000 \\
60-74 years & 0.252 & 0.186 & 0.000 \\
>74 years & 0.148 & 0.347 & 0.000 \\
Bodyweight (kg) & 0.979 & 0.003 & 0.000 \\
Diabetic Type (Type-II) & 0.617 & 0.124 & 0.000 \\
FBS & 0.997 & 0.001 & 0.000 \\
Sex (Male) & 1.79 & 0.113 & 0.000 \\
Log-like (Model) & -2061.395 & & \\
AIC & 4138.8 & & \\
\hline
\end{tabular}

In order to select variables in the model, first univariable analysis is used to check all the covariates associated with recovery time.

In this study, the predictors in the multivariable model is considered, if the test for the univariable model has a p-value less than or equal to 0.1 in the univariable analysis. Then the full multivariable Cox $\mathrm{PH}$ model is fitted including all the potential covariates which are significant at $10 \%$ at the univariable level and from multivariable model variables non-significant at $10 \%$ were eliminated using backward selection method. Accordingly variables with minimum AIC are; bodyweight at baseline, age group, sex, fasting blood sugar (FBS) at baseline and diabetic types are significant covariates selected for the model (Table 2). The multivariate results of a Cox $\mathrm{PH}$ model fitted to this dataset were obtained on Table 2. It is now observed that effects of age group, bodyweight $(\mathrm{kg})(\mathrm{p}$-value $=0.000)$ at baseline, sex $(\mathrm{p}$ value $=0.000)$, FBS $(\mathrm{mg} / \mathrm{dl})(\mathrm{p}$-value $=0.000)$ at baseline and diabetic type $(p$-value $=0.000)$ are significantly associated . Again also, the likelihood ratio test ( $\mathrm{p}$-value $=0.0001)$, Wald test $(p$-value $=0.000)$ and score $($ logrank $)$ test $(p$-value $=0.000)$ are highly significant.

\subsection{Modeling Recovery Time for Type-I and II Diabetic}

Multivariable results of a Cox PH model (1) shows that effects of age groups, bodyweight and FBS at baseline had a statistically significant impact on time-to-recovery while diastolic blood pressure is non-significant at $10 \%$ level of significance. Therefore, these variables reduced from the model.

It is now observed in the Table 3 that effects of age groups, bodyweight and FBS at baseline had a statistically significant impact on time-to-recovery. Therefore, age group, bodyweight and FBS at baseline are selected for the final model reducing the non-significant covariates. Again also, the likelihood ratio test $(\mathrm{P}$-value $=0.000)$, Wald test $(\mathrm{P}$ value $=0.000)$ and score $($ logrank $)$ test $(\mathrm{P}$-value $=0.000)$ are highly significant.

The results of a Cox PH model fitted to this dataset (TypeI) were obtained on Table 3. The hazard of the final model (2) is then given by: - 


$$
\mathrm{h}_{i}=\mathrm{h}_{0}(t) \exp \left(-0.5827 * A g e_{30-44 i}-0.61796 * A g e_{45-59 i}-2.079 * A g e_{60-74 i}-0.018 * b w_{i}-0.0028 * F B S_{i}\right)
$$

Table 3. Multivariable Cox-PH model for type-I and II DM separately.

\begin{tabular}{|c|c|c|c|c|c|c|}
\hline \multicolumn{4}{|c|}{ Cox-PH Model (Type-I DM) } & \multicolumn{3}{|c|}{ Cox-PH Model (Type-II DM) } \\
\hline Covariates & Hazard Ratio (HR) & se $(\operatorname{Coef}(\hat{\beta}))$ & P-value & Hazard Ratio(HR) & se $(\operatorname{Coef}(\hat{\beta}))$ & P-value \\
\hline \multicolumn{7}{|l|}{ Age } \\
\hline 30-44 years & 0.558 & 0.225 & 0.010 & 0.691 & 0.169 & 0.029 \\
\hline $45-59$ years & 0.539 & 0.283 & 0.030 & 0.497 & 0.164 & 0.000 \\
\hline $60-74$ years & 0.125 & 0.740 & 0.005 & 0.263 & 0.201 & 0.000 \\
\hline$>74$ & & & & 0.162 & 0.357 & 0.000 \\
\hline bodyweight (kgs) & 0.982 & 0.001 & 0.017 & 0.979 & 0.004 & 0.000 \\
\hline Sex (Male) & & & & 2.136 & 0.130 & 0.000 \\
\hline FBS (mg/dl) & 0.997 & 0.001 & 0.017 & 0.997 & 0.000 & 0.000 \\
\hline Log-like (Model) & -396.9 & & & -1457.50 & & \\
\hline AIC & 803.8 & & & 2929 & & \\
\hline
\end{tabular}

Age group, bodyweight, FBS and sex are the significant covariates associated with the recovery time of type-II diabetic mellitus whereas upper (Systolic) (110-130, Pvalue $=0.701,>130, \mathrm{P}$-value $=0.696$ ) and lower (Diastolic) $(60-80, \mathrm{P}$-value $=0.907,>80, \mathrm{P}$-value $=0.796) \mathrm{BP}$ at baseline are not significantly associated with recovery time at $10 \%$ level of significance, hence these variables not included in multivariable analysis. The results of a Cox PH model (1) fitted to this dataset were obtained on Table 3. It is observed that effects of age groups (30-44, P-value $=0.029,45-59$, Pvalue $=0.000,60-74, \mathrm{P}$-value $=0.000,>74, \mathrm{P}$-value $=0.000$ ), sex $(\mathrm{P}$-value $=0.000)$, bodyweight $(\mathrm{kg})(\mathrm{P}$-value $=0.000)$ and FBS (P-value $=0.000)$ at baseline had a statistically significant impact on time-to-recovery for type-II diabetic mellitus. A male patient recovers faster in type-II diabetic. A hazard ratio of 2.1357 corresponds to $68.11 \%$ chance of the male patient's recovered first. Rates of recovery time takes longer time in females than in male patients in type-II DM same in Cox PH.

The final Cox PH model (3) for type-II DM is then written by:-

$$
\begin{gathered}
\mathrm{h}_{i}=\mathrm{h}_{0}(t) \exp \left(-0.3694 * A g e_{30-44 i}-0.6988 * A^{2} e_{45-59 i}-1.3351 * A g e_{60-74 i}-1.821 * A g e_{>74 i}-0.0212 * b w_{i}+\right. \\
0.7588 * \text { Sex }_{i}-0.0031 * F B S_{i}
\end{gathered}
$$

In this study, in order to compare the efficiency of the models the AIC was used. The AIC is a criterion that assesses goodness of fit of a statistical model, and the lower value of AIC suggests a better model. From tables the loglikelihood and AIC values of the two types of DM that were considered as significantly influencing time to first recovery of diabetic patients.

\section{Discussion}

From the total of 544, $404(74.26 \%)$ experienced the event and the rest $140(25.74 \%)$ loss to follow-up from the study. In type-I diabetic from the total of $121,103(85.12 \%)$ experienced the event and the rest $18(14.88 \%)$ loss to follow-up and in type-II diabetic from the total of 423, 301 (71.16\%) experienced the event and the rest $122(28.84 \%)$ loss to follow-up from the study. More men than women are in diabetes; 69.42 per cent compared with 30.58 percent in those with type-I diabetes and 63.12 percent compared with 36.88 percent in those with type-II diabetes this is consistent with DM in state of UK (2011/12). The age and FBS at baseline for type-II and type-I DM are older and higher respectively as compared to cross- sectional study which was conducted in Jimma, South Western Ethiopia [13] and Mekele, Northern Ethiopia [5]. In Cox-PH model of inseparable diabetic types; age group, bodyweight, diabetic type, FBS, and sex of patients at baseline shows a statistically significant association with time to first recovery to normal blood sugar level. In univariable and multivariable analysis of Cox-PH model, the types of diabetic was a strong and independent prognostic factor, indicating better recovery time for type-I patients controlling other factors in the model. This means that patients with type-II getting affected by diabetic mellitus prolonged recovery time as compared to type-I; these findings are consistent with those done in Uganda [4]. In separate analysis of type-II DM, Cox-PH model; age group, bodyweight, FBS and sex of patients at baseline shows a statistically significant association with time to first recovery whereas in type-I DM, age group, bodyweight and fasting blood sugar of patients at baseline shows a statistically significant association with time to recovery. The results further reveal that the upper (systolic) and lower (diastolic) blood pressure has no significant effect for type-II and type-I diabetic patients, while for type-I diabetic; sex is not significantly associated. In Cox-PH model, the lower the ages, bodyweight and FBS at baseline the faster the rate of the blood sugar level returning to normal range in type-I \& II DM.

In Cox-PH model (3) the recovery time of an individual suffering from type-II diabetes who has not recovered yet has 
$0.586(\mathrm{HR}=0.586,36.95 \%$ chance of the type-II diabetic patients recovered first) times as compared to type-I DM. Being female prolonged the recovery time as compared to males $(\mathrm{HR}=2.026,66.953 \%$ chance of the male diabetic patients recovered first) in type-II DM. In Cox-PH model gender was a strong and independent prognostic factor in univariable and multivariable analysis, indicating males are better recovering to normal blood sugar as compared to females' inseparable diabetic types and type-II diabetic. This means that females getting affected by diabetic mellitus (DM) have a slightly takes longer time to recover to normal blood glucose level than males, these findings are consistent with those again obtained in Ugandan countries.

In this study, in order to compare the efficiency of models the AIC and log-likelihood were used.

\section{Conclusion}

In univariable and multivariable analysis of Cox-PH model the type of diabetic was a strong and independent prognostic factor, indicating better recovery time for type-I patients accounting and controlling other factors. This means that patients with type-II getting affected by diabetic mellitus delayed the recovery time as compared to type-I. Significant factor associated with first recovery time are important factors that should take into consideration when selecting a treatment method for both types of DM. The covariates that increased time to recovery in Cox-PH model.

Being old, female, higher FBS and overweight at baseline prolonged the recovery time; hence, concerned body should give special treatment for older, females, Higher FBS, Overweight, and type-II DM of patients at baseline. I will recommend the researchers as advanced statistical models should be used to account extra variability that accounts time to recovery of types of DM.

\section{Acknowledgements}

My grateful thanks to my parents, whose love, trust, encouragement, comfort and support always surround. I am grateful to all the faculty, department, and friends who ever helped. Lastly not from least, I would like to thank Jimma University Specialized Hospital (JUSH) Diabetic Patient Clinic for their great help in many ways during data collection.

\section{Conflict of Interest Statement}

All the authors do not have any possible conflicts of interest.

\section{References}

[1] Leong, W. Y. Survival analysis of Diabetic patients in queen Elizabeth hospital, kotan kinabalu. 2007.

[2] Connor, H. and Boulton, A. J. M. (1989). Diabetes in Practice. Chichester: Wiley.

[3] Guariguata, L., Whiting, D. R., Hambleton, I., Beagley, J., Linnenkamp, U., \& Shaw, J. E. Global estimates of diabetes prevalence for 2013 and projections for 2035 for the IDF Diabetes Atlas. Diabetes Research and Clinical Practice, 2013.

[4] Olive D, Odwee $\mathrm{O}$ and Atuhaire $\mathrm{K}$,. The determinants of recovery time of diabetic patients from three hospitals in Uganda, 2007.

[5] Gebregziabher, M., Egede, LE., Lynch, CP., Echols, C., Zhao, Y. (2010). Effect of Trajectories of Glycemic Control on Mortality in Type II Diabetes.

[6] Dereje, A. (2005). Diabetes Mellitus, Diploma program for the Ethiopian health center team.

[7] Kaplan and Meier. Non-parametric estimation from incomplete observations. Journal of American Statistical Association, 1958.

[8] Cox, D. Regression models and life tables (with discussion) Journal of the Royal Statistical Society B, 1972.

[9] Green, P. J. (1987). Penalized likelihood for general semiparametric regression models. International Statistical Review.

[10] Schoenfeld, D. (1982). Partial residuals for the proportional hazards regression model.

[11] Hosmer DW. \& Lemeshow. Applied survival analysis regression modeling of time to event data, 1999.

[12] Collet, D,. Modeling Survival Data in medical research second edition, 1972.

[13] Endalew, H., H. Mariam, W., Belachew, T., Birhanu, Z. (2012). Self-care practice and glycaemic control amongst adults with diabetes at the Jimma University Specialized Hospital in south-west Ethiopia: A cross-sectional study. 